\title{
Novos Tempos, Novos Desafios: Estratégias para Equidade de Acesso ao Ensino Remoto Emergencial
}

\author{
New Times, New Challenges: Strategies to Ensure Equal Access to Emergency Remote Education
}

Simone Appenzeller' (D), Fábio Husemann Menezes' (D), Gislaine Goulart dos Santos' (iD Roberto Ferreira Padilha' (D, Higor Sabino Graça' (D), Joana Fróes Bragança' (D)

\begin{abstract}
RESUMO
Introdução: Com a suspensão das atividades presenciais e a implementação do ensino remoto do curso de Medicina da Unicamp, foi necessária a elaboração de estratégias para identificar as necessidades dos estudantes para continuidade das aulas nessa nova modalidade.

Objetivo: Demonstrar as estratégias desenvolvidas e as soluções encontradas para permitir a equidade de acesso ao ensino remoto no curso de medicina da FCM-Unicamp.
\end{abstract}

Método: São apresentados dados do relatório institucional, elaborado a partir de questionários aplicados para os estudantes do curso de Medicina sobre as dificuldades referentes ao acesso ao ensino remoto emergencial e as ações realizadas para solucionar as principais dificuldades encontradas.

Resultados: Constatamos que os anos iniciais do curso apresentam a maior proporção de alunos com dificuldades, embora, em todos os anos, os discentes tenham relatado alguma dificuldade. Os principais problemas identificados foram internet instável e/ou apenas acesso por redes móveis. Verificou-se ainda que os alunos tinham maior dificuldade em acompanhar as atividades síncronas. A maior parte dos alunos mencionou que acompanhava as aulas por computadores e notebooks, mas, muitas vezes, estes eram compartilhados com outros membros da família. Alguns estudantes apontaram também que muitas vezes, não conseguiam acompanhar o curso por falta de aparelhos ou acesso à internet. Nesse caso, computadores e chips de celular e para uso em tablets eram emprestados aos alunos. Houve orientação aos professores para adequação das ferramentas pedagógicas utilizadas.

Conclusão: Garantir a equidade de acesso é fundamental para permitir a continuidade dos estudos na transformação do estudo presencial em remoto emergencial. $O$ ato de escutar os alunos sobre as dificuldades em relação ao ensino remoto e a realização de suporte material são ferramentas essenciais para o sucesso dessa estratégia pedagógica. A orientação do corpo docente em relação às dificuldades dos alunos foi importante para adequação do ensino remoto. Essas ações auxiliaram os estudantes na mudança do ensino imposta pelo distanciamento social.

Palavras-chave: Materiais de Ensino; Equidade; Educação Médica.

\begin{abstract}
Introduction: After the implementation of remote teaching for the academic activities of the Medicine course at UNICAMP, due to the COVID Pandemic, a new strategy was required to identify the needs of students to allow them to keep up with the course in this new pedagogical modality.

Objective: To demonstrate the strategies developed and solutions found to allow for equal access to remote education in the medical course at FCM-UNICAMP.

Method: We present data from our institutional report based on questionnaires completed by medical students regarding the difficulties related to access to emergency remote education and the actions taken to solve the main difficulties encountered.

Results: We identified that the initial years of the course had the highest proportion of students with difficulties, although all students reported some difficulty. The main problems identified were unstable internet and/or only access via mobile networks, and the most significant difficulty was in accessing the synchronous activities. Most students reported attending classes via computer and notebooks, but this device was often shared with other family members, most notably during the pandemic. Thirty-one students reported difficulties that prevented them from following the course adequately due to lack of devices or internet access. One solution was computers and cell phone chips and tablets being loaned out to students. Teachers were instructed to adapt the pedagogical tools used.
\end{abstract}

Conclusion: Ensuring equal access is essential to support studies to continue following the transition from face-to-face classes to emergency remote study. Listening to students about the difficulties concerning remote teaching is an essential tool for the success of this pedagogical strategy. Guidance of the teaching staff in relation to the difficulties encountered by students was important for the adequacy of remote teaching. These actions helped students in the change of teaching imposed by social distancing.

Keywords: Teaching Materials; Equal Opportunities; Medical Education.

'Universidade Estadual de Campinas, Campinas, São Paulo, Brasil.

Correspondência:

Simone Appenzeller.

Universidade Estadual de Campinas, Rua Tessália Vieira de Camargo, 126, Cidade Universitária Zeferino Vaz, Campinas, SP, Brasil. CEP: $13083-887$.

E-mail: appenzel@unicamp.br

Recebido em 11/08/20; Aceito em 12/09/20 


\section{INTRODUÇÃO}

A pandemia da síndrome respiratória aguda grave do coronavírus 2 (severe acute respiratory syndrome coronavirus 2 - Sars-CoV-2) apresenta um impacto mundial na população, na economia e, principalmente, nos serviços de saúde. As consequências em curto prazo são muitas vezes devastadoras, porém pouco se sabe sobre as consequências em médio e longo prazos. O distanciamento social, além das medidas de higiene e uso de máscara, tornou-se um dos pilares principais para evitar a sobrecarga do sistema de saúde ${ }^{1,2}$. Com isso, logo no início da disseminação viral, houve discussão sobre a suspensão de aulas em todas as esferas da educação. A Universidade Estadual de Campinas (Unicamp) optou pela suspensão do ensino presencial em março 2020, como em muitas outras universidades do mundo, o que gerou novas demandas para o ensino ${ }^{3}$. Essa suspensão foi acompanhada por busca de métodos e tecnologias inovadores de ensino como relatado em outras instituições ${ }^{4}$. A Faculdade de Ciências Médicas (FCM-Unicamp) optou pelo ensino remoto emergencial, utilizando majoritariamente a plataforma Google Sala de Aula'.

O uso de novas estratégias pedagógicas trouxe desafios, como capacitação docente, adaptação dos estudantes, saúde mental da comunidade e manejo do tempo para estudo, e a garantia de acesso por parte dos estudantes tornou-se uma preocupação da comunidade acadêmica5 .

A Unicamp tem, desde o vestibular de 2005, o Programa de Ação Afirmativa e Inclusão Social (Paais), cujo objetivo é incluir alunos egressos do ensino médio da rede pública. Esse programa foi modificado ao longo dos anos, de modo a permitir um perfil sociocultural mais abrangente no ingresso de alunos ${ }^{6}$. Em 2018, acrescentou-se a esse programa uma bonificação para raça (negros, pardos e indígenas). Com isso, observou-se uma maior porcentagem de alunos pardos e negros e oriundos de escolas públicas. Na Medicina, esse percentual chegou a $85 \%$ no vestibular de 20197 . Também se observou uma mudança no perfil socioeconômico, com predominância nos estratos $\mathrm{C}_{1}$ e B27.

Atualmente o curso de Medicina apresenta maior pluralidade social, cultural e de saberes. Entretanto, a permanência estudantil tornou-se um maior desafio com as novas demandas acolhidas. A universidade oferece várias opções de auxílio para garantir a permanência estudantil, como bolsa auxílio-trabalho (BAS), auxílio-alimentação, moradia, além do incentivo a atividades de pesquisa com bolsas desde os anos iniciais.

A escolha do ensino remoto, associada a essa mudança no perfil socioeconômico do curso de Medicina, enfatizou a necessidade de escuta acurada dos alunos. Foram necessárias ações institucionais com o intuito de garantir a equidade de acesso e o acompanhamento do conteúdo, inicialmente teórico. Além disso, houve a necessidade de readequar o acesso a bolsas-trabalho utilizando tecnologias remotas para a manutenção dos auxílios para quem necessita.

O objetivo deste trabalho é demonstrar as estratégias desenvolvidas e soluções institucionais encontradas para promover a equidade de acesso ao ensino remoto no curso de Medicina da FCM-Unicamp.

\section{MÉTODO}

Após a suspensão das atividades presenciais, foi criado prontamente pela gestão um grupo composto de professores e funcionários da comissão de ensino da FCM-Unicamp para dar suporte às necessidades da comunidade em relação ao ensino remoto emergencial.

Um dos primeiro objetivo deste grupo foi de mapear as necessidades dos estudantes em relação ao ensino remoto para avaliação das dificuldades discentes em relação ao acompanhamento do ensino remoto emergencial. O primeiro levantamento foi realizado em 23 de março de 2020, dez dias após a suspensão das atividades presenciais. Encaminhouse para todos os estudantes do curso de Medicina da FCMUnicamp, por meio do e-mail institucional e do grupo de WhatsApp criado pela coordenação do curso, um questionário elaborado no Google Forms. Por meio desse grupo e do contato estreito com os representantes discentes, foi estimulado o preenchimento do formulário. O questionário incluía as seguintes informações: nome, registro acadêmico, cidade em que o aluno mora durante o período de suspensão das atividades presenciais, se é bolsista do serviço de apoio ao estudante (SAE), acompanhamento das aulas em ensino remoto, tipo e qualidade de acesso à internet, equipamento disponível para acompanhamento das aulas em ensino remoto, compartilhamento do principal equipamento de estudo com membros da casa, dificuldade(s) no uso ou na falta desses equipamentos para o estudo, dificuldades encontradas em acompanhar disciplinas e motivo. O resultado desse questionário orientou a atuação da coordenação de ensino para garantir o acesso de todos os estudantes do curso.

Um mês após a implementação do ensino remoto emergencial na instituição, fez-se um novo levantamento por questionário elaborado no Google Forms que foi enviado a todos os estudantes do curso. A proposta desse levantamento era avaliar as atividades em ensino remoto e identificar os formatos de ensino de conteúdo teórico com maior facilidade de acompanhamento por parte dos alunos. O objetivo desse questionário foi permitir o aprimoramento das atividades realizadas e adequar os conteúdos ao tipo de acesso utilizado pela maioria dos estudantes. 
Por fim, um terceiro levantamento institucional foi realizado, após quatro meses de ensino remoto emergencial, que analisou o uso das plataformas Google Classroom e do canal do YouTube. Esse levantamento referia-se aos conteúdos ministrados e tinha como objetivo avaliar a quantidade de material postado e a forma e quantidade de acesso dos alunos. Durante todo período, a coordenação manteve contato próximo com os estudantes, gestores e professores para verificar dificuldades individuais e coletivas e realizou intervenções imediatas, quando isso fosse necessário.

Os resultados foram compilados em planilhas Excel. Os dados são apresentados de forma descritivas.

\section{RESULTADO}

$\mathrm{Na}$ primeira enquete, obtiveram-se 563 respostas de um total de $729(77,22 \%)$ estudantes matriculados. O terceiro ano teve o menor índice de respostas, seguido pelo segundo e sexto anos (Tabela 1). Apesar da possibilidade, observamos que nenhum aluno optou por trancar o semestre até a presente data. A maioria dos estudantes acessavam as aulas de ensino remoto por computador, notebook ou celular (80\%). Acesso exclusivo por celular foi referido por 10\%, e 10\% mencionaram tablet.

Os principais problemas identificados foram internet instável e/ou acesso exclusivo por redes móveis. As atividades com maior dificuldade de acompanhamento pelos alunos eram transmitidas por webconferências e meetings virtuais, seguidos por acesso a plataformas digitais e aplicativos de imagem. Identificamos que os anos iniciais do curso apresentavam a maior proporção de alunos com dificuldades de acesso. A maior parte dos alunos referiu que acompanhava as aulas por computadores $\mathrm{e}$ notebooks, mas muitas vezes esses aparelhos eram compartilhados com outros membros da família. Compartilhamento do principal equipamento de estudo com outro membro da família foi referido por $10 \%$ a $48 \%$ dos respondentes.

Tabela 1. Dados demográficos, auxílios e tipo de internet dos respondentes de acordo com o ano do curso de Medicina

\begin{tabular}{ccccc}
\hline & & \multicolumn{3}{c}{ Tipo de internet } \\
\hline Ano & $\begin{array}{c}\text { Número de } \\
\text { respondentes }\end{array}$ & $\begin{array}{c}\text { Apenas } \\
\text { Wi-fi }\end{array}$ & $\begin{array}{c}\text { Apenas } \\
\text { rede móvel }\end{array}$ & $\begin{array}{c}\text { Wi-fi e rede } \\
\text { móvel }\end{array}$ \\
\hline $1^{\circ}$ & $107 / 121(88,4 \%)$ & 44 & 8 & 55 \\
\hline $2^{\circ}$ & $85 / 120(70,8 \%)$ & 34 & 4 & 45 \\
\hline $3^{\circ}$ & $85 / 126(67,4 \%)$ & 18 & 0 & 66 \\
\hline $4^{\circ}$ & $115 / 130(88,4 \%)$ & 36 & 3 & 75 \\
\hline $5^{\circ}$ & $91 / 118(77,1 \%)$ & 29 & 0 & 61 \\
\hline $6^{\circ}$ & $80 / 114(70,2 \%)$ & 13 & 1 & 64 \\
\hline
\end{tabular}

Fonte: Elaborada pelos autores.
Com base nesses resultados, foi realizado um levantamento de necessidades estudantis referente à disponibilidadedecomputadoresouacessodeinternetadequada para acompanhamento do estudo remoto emergencial. Com a reitoria viabilizou-se o empréstimo de computador/tablet e chip de celular para acesso da internet que foram disponibilizados aos alunos. Quando contactados, aproximadamente 50\% dos alunos que solicitaram compareceram para retirada dos equipamentos. O restante desistiu dos empréstimos referindo resolução da dificuldade de acesso por outros meios.

Após quatro semanas de ensino remoto emergencial, fez-se um segundo levantamento para identificar as melhores ferramentas utilizadas pela avaliação dos estudantes. Nessa pesquisa, obtivemos $419 / 729$ (57,5\%) respostas. Constatou-se que as aulas assíncronas, postadas em plataforma de streaming, tinham maior facilidade de acesso e aproveitamento pelos alunos, seguidas por estudos dirigidos (Gráfico 1). Estudantes referiam dificuldades para acompanhar aulas síncronas. Observou-se que o acesso principal ainda era realizado por computador, seguido por dispositivos móveis (Gráfico 2).

Após quatro meses de ensino remoto emergencial, estavam disponíveis para os alunos 442 videoaulas para o curso de Medicina. A distribuição por turma está demonstrada no Gráfico 3. O reduzido material postado nos primeiros dois anos deve-se ao fato de que a maioria das disciplinas nesses anos é oferecida pelo Instituto de Biologia que possui seu próprio canal de streaming e gerenciamento de conteúdo. No internato, por ser um período de atividades principalmente práticas, também observamos uma redução de videoaulas postadas. As atividades realizadas para os quinto e sexto anos foram majoritariamente visitas virtuais, discussões de casos clínicos e seminários online.

Gráfico 1. Avaliação dos alunos em relação ao formato para acompanhamento das aulas do estudo remoto emergencial após quatro semanas de implementação

Qual o melhor formato para acompanhamento das aulas? (3 opções)
419 respondentes

$$
419 \text { respondentes }
$$

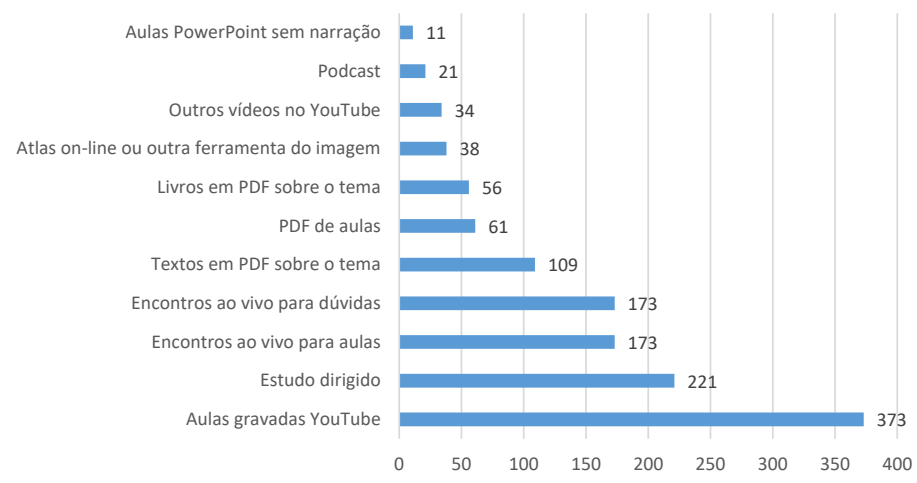

Fonte: Elaborado pelos autores. 
Gráfico 2. Dispositivos utilizados pelos alunos para assitir às aulas do curso em período de ensino remoto

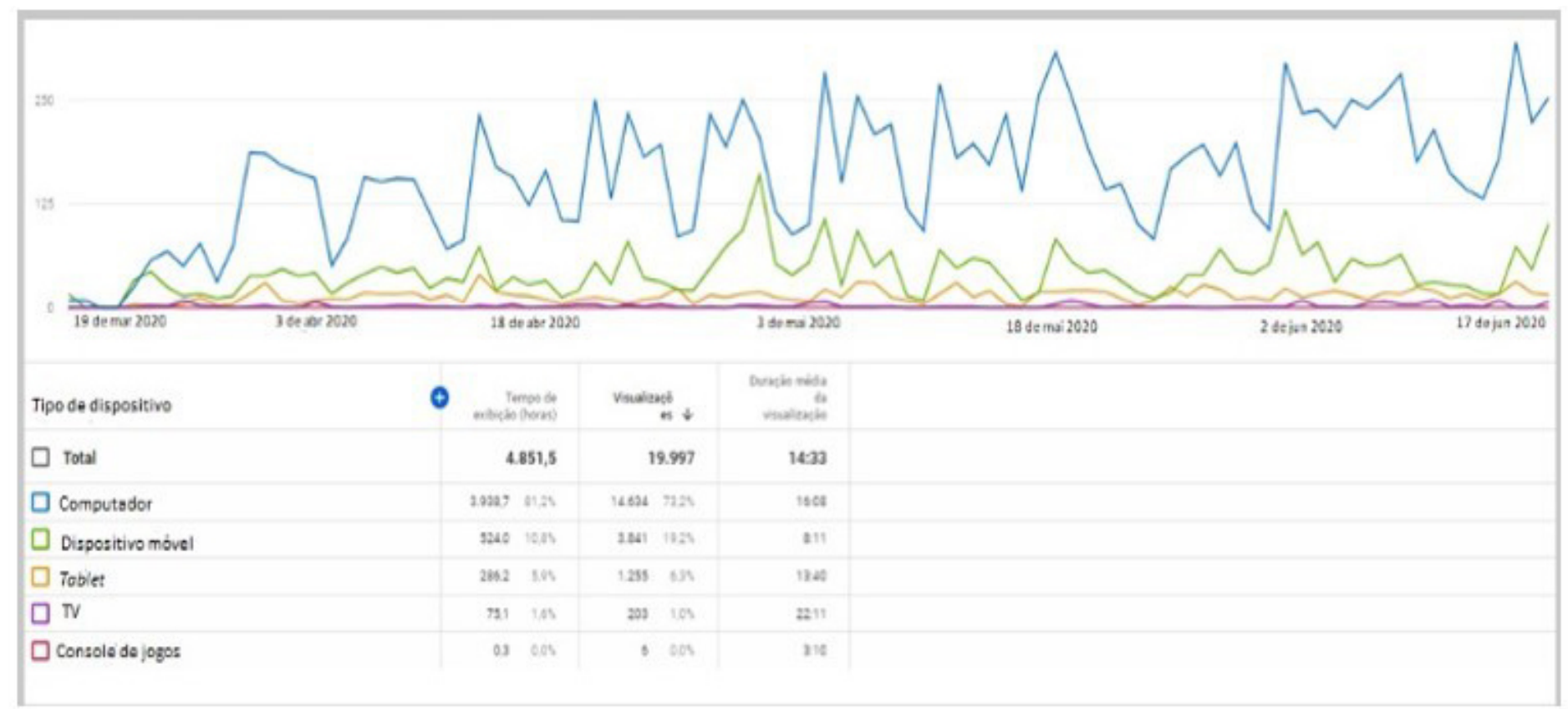

Fonte: Elaborado pelos autores.

\section{DISCUSSÃO}

Garantir a equidade de acesso é fator fundamental para permitir a continuidade do processo ensino-aprendizagem na transformação do estudo presencial em remoto emergencial. A coordenação do curso de Medicina elaborou estratégias contínuas ao longo desses quatro meses para identificar necessidades e solucionar problemas, principalmente relacionados à equidade.

Escutar os alunos sobre as dificuldades em relação ao ensino remoto emergencial foi ferramenta essencial para o sucesso dessa estratégia pedagógica. Além dos grupos de WhatsApp e mensagens eletrônicas, a procura ativa por meio de questionários foi um pilar fundamental para acompanhar e identificar precocemente as dificuldades dos estudantes. A experiência de protagonismo dos alunos na atuação em relação ao ensino remoto emergencial é fator determinante para o sucesso do ensino nos tempos de pandemia ${ }^{8}$. Observamos $77 \%$ de respondentes no primeiro questionário e $56 \%$ no segundo questionário, embora tenha sido estimulada a resposta por parte dos estudantes por vários meios de comunicação. A taxa de resposta é elevada quando comparada com a taxa de resposta observada na literatura9. Embora o próprio acesso ao questionário possa ter sido um fator excludente, o acompanhamento do acesso desses estudantes ao material postado e a atenção contínua de gestores, professores e representação discente foram estratégias para identificar a ausência de alunos nas atividades e dificuldades relativas ao curso.

O auxílio material da Pró-Reitoria de Graduação para garantir computadores e redes de celulares aos alunos foi de extrema importância para a acessibilidade e a equidade de acesso
Gráfico 3. Número de videoaulas disponíveis para os alunos de Medicina da FCM-Unicamp por ano do curso, em um período de quatro meses $(n=442)$

Número de videoaulas no YouTube por ano (disciplinas MD)

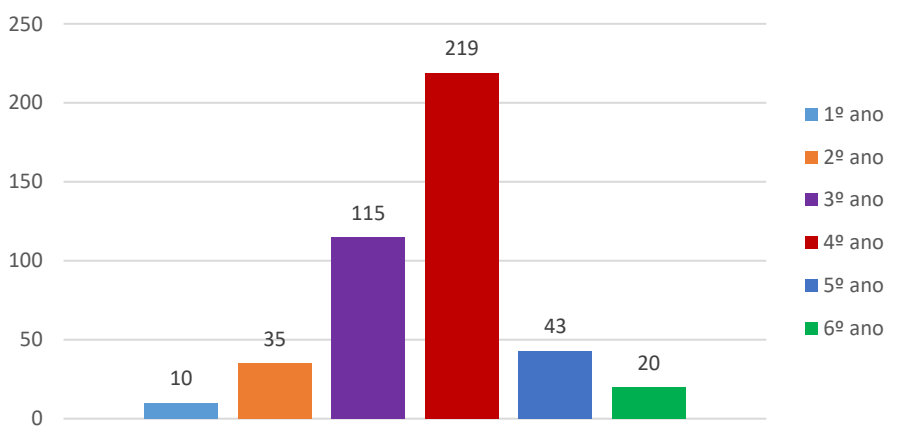

MD: Medicina.

Fonte: Elaborado pelos autores.

ao ensino remoto emergencial. Por meio de questionários, fomos capazes de acompanhar os discentes e identificar necessidades materiais precocemente. Com isso, observamos que nenhum aluno trancou o semestre ou ano, embora eles tivessem a oportunidade sem ônus para a integralização do curso, por conta da política de excepcionalidade instituída pela universidade neste momento de pandemia.

Ensino remoto emergencial é caracterizado pela mudança temporária do ensino presencial para o ensino remoto. O ensino passa, em um momento de crise, como no caso da pandemia da Sars-CoV-2, para totalmente remoto, e todas as orientações e todo o conteúdo educacional são 
ministrados em plataformas a distância. $O$ objetivo educacional não é criar um curso a distância robusto, mas fornecer acesso temporário à instrução e apoio instrucional de uma maneira que seja rápida de configurar e que esteja disponível de forma confiável durante o período. É importante ressaltar que o ensino remoto emergencial apresenta diferenças fundamentais dos modelos de ensino a distância ou modelo híbrido que têm um planejamento prévio de conteúdo e tempo cuidadoso usando modelos de desenvolvimento e planejamento bem conhecidos $^{10,11}$. O entendimento dessas diferenças é fundamental para reduzir a ansiedade por parte dos docentes, que não se sentem preparados para ministrar aulas não presenciais, e dos discentes. Porém, como nos programas presenciais, a avaliação contínua do programa é a principal ferramenta de aprimoramento. No nosso caso, a estratégia de implementação de grupo para acompanhamento das atividades de ensino e contato estreito com estudantes para diagnóstico precoce das necessidades discentes e docentes e adequação contínua da estratégia pedagógica foi exitosa com grande produção de material de ensino, acolhimento dos alunos e manutenção dos estudantes nas atividades sem nenhum caso de desistência ou trancamento do curso.

A capacitação do corpo docente também é importante fator para o sucesso do ensino remoto. A coordenação de ensino realizou capacitação inicial de forma rápida para poder dar início às atividades remotas. Elaborou um manual de orientação para o ensino remoto que contemplava técnica de gravação e disponibilização de aulas e como postar e organizar o material na plataforma. Deu suporte inicialmente presencial e depois a distância aos professores que apresentavam dificuldades com alguma das etapas. Além disso, a equipe da coordenação do curso de Medicina manteve verificação, de forma rotineira, do material postado para identificar problemas de organização e sobrecarga de conteúdo, assim como a adequação do material com os objetivos pedagógicos das disciplinas.

Em um momento inicial, a maior dificuldade encontrada para a estruturação do curso em modelo remoto foi a inserção de conteúdo excessivo e de forma desorganizada nas plataformas. Os professores foram encorajados a avaliar cuidadosamente os conteúdos postados, orientar com clareza o objetivo de aprendizagem de cada aula e adequálos de acordo com as competências previamente atribuídas à disciplina. Recomendou-se fortemente a utilização de metodologia assíncrona. Nos encontros síncronos, solicitouse a gravação das reuniões com posterior disponibilização, identificação e suporte dos estudantes com dificuldades de acesso. Encontros para trocas e resolução de dúvidas entre docentes/coordenadores de disciplinas e alunos também foram organizados. A coordenação auxiliou na organização do material dividindo-os em conteúdos essenciais e complementares. Esse processo foi cuidadosamente monitorado ao longo dos meses.

Como complementação desse processo, houve um dia de avaliação do curso no modelo virtual com a participação ativa dos discentes. Foi um importante momento de troca entre docentes e discentes e de escuta e acolhida das dificuldades e ansiedades dos alunos em relação ao período de isolamento social, pandemia e mudança de estratégia pedagógica. Esse encontro trouxe novas informações e sistematizou as já existentes, ajudando ainda mais as ações de aprimoramento do ensino.

Ao longo dos meses, observamos que não houve diminuição do número de acessos ao conteúdo postado.

\section{CONCLUSÃO}

A identificação de estudantes com dificuldade de acesso, a escuta atenta sobre o ensino remoto e a doação de dispositivos permitiram reduzir a inequidade entre os discentes em relação ao acesso às plataformas remotas, de modo a evitar que essa estratégia pedagógica fosse um fator de desigualdade no processo ensino-aprendizagem. A atuação para diagnóstico de forma imediata pela equipe da coordenação de ensino de graduação com suporte da próreitoria permitiu auxiliar os estudantes na mudança do ensino imposta pelo distanciamento social. A capacitação inicial do corpo docente para o manejo ferramentas pedagógicas virtuais e a informação constante sobre as dificuldades encontradas pelos alunos em relação às ferramentas utilizadas foram fatores extremamente importantes para a adequação do ensino remoto emergencial.

\section{CONTRIBUIÇÃO DOS AUTORES}

Simone Appenzeller, Fábio Husemann Menezes, Gislaine Goulart dos Santos, Roberto Ferreira Padilha, Higor Sabino Graça e Joana Fróes Bragança participaram da elaboração do projeto, da aquisição de dados e da aprovação para publicação. Simone Appenzeller e Joana Fróes Bragança participaram da escrita do artigo.

\section{CONFLITO DE INTERESSES}

Os autores declaram não haver conflito de interesses neste estudo.

\section{REFERÊNCIAS}

1. Al Samaraee A. The impact of the COVID-19 pandemic on medical education. Br J Hosp Med (Lond). 2020;81(7):1-4.

2. Sandhu $P$, de Wolf $M$. The impact of COVID-19 on the undergraduate medical curriculum. Med Educ Online. 2020;25(1):1764740. 
3. Burki TK. COVID-19: consequences for higher education. Lancet Oncol. 2020; $21(6): 758$.

4. Woolliscroft JO. Innovation in response to the COVID-19 pandemic crisis. Acad Med. 2020;95(8):1140-2.

5. Rajab MH, Gazal AM, Alkattan K. Challenges to online medical education during the COVID-19 pandemic. Cureus. 2020;12(7):e8966.

6. Tessler L, Pedrosa R. PAAIS: a experiência de um programa de ação afirmativa na Unicamp. Movimento em Debate - Adunicamp. 2008;1(2):9.

7. Silva MLAM, Amaral E, Machado HC, Passeri SMRR, Bragança JF. Influência de políticas de ação afirmativa no perfil sociodemográfico de estudantes de Medicina de universidade brasileira. Rev Bras Educ Med. 2018;42(3):36-48.
8. Theoret $\mathrm{V}$, Ming $\mathrm{X}$. Our education, our concerns: the impact on medical student education of COVID-19. Med Educ. 2020;54(7):591-2.

9. Nulty DD. The adequacy of response rates to online and paper surveys: what can be done? Assessment \& Evaluation in Higher Education. 2008;33(3):301-14.

10. Bernard RM, Abrami PC, Borokhovski E, Wade AC, Tamim RM, Surkes $M A$, et al. A meta-analysis of three types of interaction treatments in distance education. Rev Educ Res. 2009; 79(3):1243-89.

11. Hodges C, Moore S, Lockee B, Trust T, Bond A. The difference between emergency remote teaching and online learning [acesso em 6 jun 2020]. Disponível em: https://er.educause.edu/articles/2020/3/the-differencebetween-emergency-remote-teaching-and-online-learning. 